\title{
Imaging the Phase Transformation in Single Particles of the Lithium Titanate Anode for Lithium-Ion Batteries
}

\author{
Tadesse A. Assefa,* Ana F. Suzana, Longlong Wu, Robert J. Koch, Luxi Li, Wonsuk Cha, Ross J. Harder, \\ Emil S. Bozin, Feng Wang, and Ian K. Robinson*
}

Cite This: https://dx.doi.org/10.1021/acsaem.0c02010

Read Online

ACCESS

Џll Metrics \& More

回 Article Recommendations

(s) Supporting Information

ABSTRACT: Lithium uptake and release in lithium titanate (LTO) anode materials during a discharge and charge cycle is one of the fundamental processes of a lithium-ion battery (LIB), still not fully understood at the microscopic level. During the discharge cycle, LTO undergoes a phase transformation between $\mathrm{Li}_{4} \mathrm{Ti}_{5} \mathrm{O}_{12}$ and $\mathrm{Li}_{7} \mathrm{Ti}_{5} \mathrm{O}_{12}$ states within a cubic crystal lattice. To reveal the details of the microscopic mechanism, it is necessary to track the sequence of phase transformations at different discharge/charge states under operating conditions. Here, we use in situ Bragg coherent diffraction imaging (BCDI) and in situ X-ray diffraction (XRD) experiments

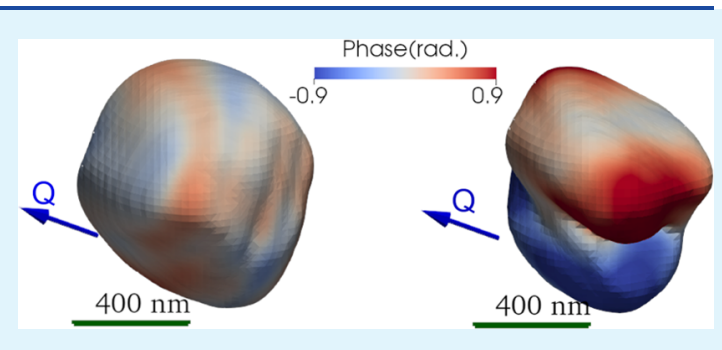
to examine the lithium insertion-induced materials phase transformation within a single LTO particle and a bulk battery analogue, respectively. BCDI analysis from (111) Bragg peak shows the two-phase transformation manifesting as a distinct image phase modulation within a single LTO nanoparticle occurring in the middle of the discharge region then subsiding toward the end of the discharge cycle. We observe the biggest phase variation at the two-phase stage, indicating the formation of phase domains of 200 nm in size during the discharge process. We also observe a lattice contraction of $>0.2 \%$ in a single LTO nanoparticle at the (400) Bragg peak measurement, larger than that in the corresponding bulk material. Our observation of this phase transformation at a singleparticle level has implications for the understanding of the microscopic/mesoscale picture of the phase transformation in anode and cathode LIBs materials.

KEYWORDS: coherent diffraction imaging, image phase variation, displacement field, battery material, lithium titanate anode

\section{INTRODUCTION}

As the world population grows, its demand for energy grows, leading to the depletion of nonrenewable energy sources. The use of these energy sources leads to a detrimental impact on the environment, and this leads to the search for clean, renewable energy sources. Solar energy is the most abundant source of energy, but much of it is not used due to the lack of efficient energy-storage devices. Tremendous progress has been made in search and developing electrochemical storage devices; one example is rechargeable lithium-ion batteries (LIBs), which show excellent cycling stability and high-rate capability. $^{1-3}$ The performance of LIBs depends on the cathode, anode, and electrolyte/binder choices, ${ }^{4}$ which can be optimized for different applications. In LIBs, the cathode electrode is the source of $\mathrm{Li}^{+}$ions, while the electrolyte serves as a conductive medium that helps $\mathrm{Li}^{+}$ion and electron transport, the separator prevents direct contact between anode and cathode electrodes, and the anode electrode stores and releases $\mathrm{Li}^{+}$ions from the cathode. ${ }^{4}$ Since the introduction of the first commercialized rechargeable Li-ion battery in 1991 by Sony, different anode and cathode materials have been explored to improve the power density, cyclability, and safety of the battery. ${ }^{5}$ Cathodes such as $\mathrm{LiFePO}_{4}$ and $\mathrm{LiCoO}_{2}$ and anodes such as $\mathrm{Li}$, graphite (by forming intercalation compound $\mathrm{LiC}_{6}$ ), $\mathrm{Si}$, and $\mathrm{Li}_{4} \mathrm{Ti}_{5} \mathrm{O}_{12}$ have been used in different combinations. ${ }^{6} \mathrm{Li}$ metal has been used as the anode material that could provide high specific capacity $(3860 \mathrm{mAh} / \mathrm{g})$ and lowest overall anode potential. However, cycling of the $\mathrm{Li}$ anode leads to the formation of dendrites, which could eventually penetrate the separator and cause a shortage and fire in the battery. ${ }^{7-9}$ Also, graphite has high energy density and long cycle life, which makes it the most popular anode in commercial LIBs, but the low lithiation voltage $(\sim 0.1 \mathrm{~V}$ vs $\mathrm{Li} /$ $\mathrm{Li}^{+}$) can result in a short circuit and possibly fire. Although a $\mathrm{Si}$ anode has the highest known theoretical capacity (4200 mAh/ $\mathrm{g}$ ), its huge volume expansion, over $300 \%$, occurring during the lithiation/delithiation cycles leads to electrical shorting/ disconnection between the active materials and the current collector. ${ }^{5,10-12} \mathrm{Li}$, graphite, and $\mathrm{Si}$ anodes have failed so far to satisfy the fast-charging capabilities in LIBs. In contrast, $\mathrm{Li}_{4} \mathrm{Ti}_{5} \mathrm{O}_{12}$ (lithium titanate or LTO) has long-cycling stability

Received: August 19, 2020

Accepted: December 11, 2020 
and high-rate capability because it has insignificant volume change $(<0.2 \%)$ between fully lithiated and delithiated endmembers; ${ }^{13-15}$ this makes LTO a promising anode material for use in commercial fast-charging batteries, such as Toshiba's $\mathrm{SCiB}$ rechargeable batteries. ${ }^{16}$

$\mathrm{Li}_{4} \mathrm{Ti}_{5} \mathrm{O}_{12}$ and $\mathrm{Li}_{7} \mathrm{Ti}_{5} \mathrm{O}_{12}$ are two end-members with a defective spinel (space group $F d \overline{3} m$ ) and rock-salt (space group $F m \overline{3} m$ ) structure, respectively. ${ }^{17-19}$ The crystal structure of LTO has cubic symmetry with lattice constants of $a=b=c$ $=8.3595 \AA$ and $\alpha=\beta=\gamma=90^{\circ}$. When LTO is fully discharged, three $\mathrm{Li}$ ions are inserted per unit cell, forming $\mathrm{Li}_{7} \mathrm{Ti}_{5} \mathrm{O}_{12}$ with lattice constants of $a=b=c=8.3538 \AA$ and $\alpha$ $=\beta=\gamma=90^{\circ 17}$ showing a $0.07 \%$ lattice contraction. Compared to $\mathrm{Li}_{4} \mathrm{Ti}_{5} \mathrm{O}_{12}$, the octahedral (16c) positions in $\mathrm{Li}_{7} \mathrm{Ti}_{5} \mathrm{O}_{12}$ are occupied by $\mathrm{Li}$, and the octahedral (16d) sites maintain the same configuration (occupation by $\mathrm{Li}$ and $\mathrm{Ti}$ ). The electrochemical reaction in LTO proceeds via two-phase transformation between $\mathrm{Li}_{4} \mathrm{Ti}_{5} \mathrm{O}_{12}$ (with the octahedral (16d) sites randomly occupied by $\mathrm{Li}$ or $\mathrm{Ti}$ atoms and the tetrahedral $(8 \mathrm{a})$ positions occupied by $\mathrm{Li}$ atoms) and $\mathrm{Li}_{7} \mathrm{Ti}_{5} \mathrm{O}_{12}$ (the octahedral (16c) positions occupied by $\mathrm{Li}$ atoms and the occupancy of the octahedral (16d) identical to that in $\mathrm{Li}_{4} \mathrm{Ti}_{5} \mathrm{O}_{12}$ ) end-member LTO phases. ${ }^{17}$ During $\mathrm{Li}^{+}$uptake, the Li-ions at the $8 \mathrm{a}$ sites move to the empty $16 \mathrm{c}$ sites, and the new $\mathrm{Li}$ ions from the electrolyte also occupy the $16 \mathrm{c}$ sites. In the fully lithiated state, three additional $\mathrm{Li}^{+}$ions are added per unit cell. However, visualization of structural phase transformation and tracking the associated lithium migration directly with $\mathrm{X}$-ray-based techniques is experimentally challenging since the lattice constant change between the two end-members is very small $(<0.1 \%)$, in addition to the low atomic scattering factor of $\mathrm{Li}$ compared to that of $\mathrm{O}$ and $\mathrm{Ti}$ atoms. Different experimental techniques such as X-ray absorption spectroscopy, ${ }^{20}$ electron energy-loss spectroscopy (EELS), ${ }^{21}$ neutron powder diffraction, ${ }^{22}$ and nuclear magnetic resonance (NMR) ${ }^{23-25}$ have been implemented to identify the structural phase transformation in LTO anode materials. A $\mathrm{Ti} \mathrm{K}$ edge X-ray absorption spectroscopy study supported by ab initio calculations by Zhang et al. proposed a new quasi solidsolution phase, which plays an instrumental role in facilitating $\mathrm{Li}^{+}$transport. Using STEM-EELS spectrum imaging of the lithiated $\mathrm{Li}_{7} \mathrm{Ti}_{5} \mathrm{O}_{12}$, Kitta et al. showed that the two separate phases coexist inside the specimen with defined phase boundaries, and no apparent misfit strains or misorientations are detected. ${ }^{26}$ Monte-Carlo simulation combined with density functional theory (DFT) on a large LTO system showed that the three energetic parameters, namely, the potential energy of the $8 \mathrm{a}$ site, the difference in the site energy between the $8 \mathrm{a}$ and $16 \mathrm{c}$ sites, and the repulsion between two $\mathrm{Li}$ atoms situated at the adjacent $8 \mathrm{a}$ and $16 \mathrm{c}$ sites and the topology of the Li sites, determine the system. ${ }^{27}$ For the electrochemical potential profile, the differences in the site energy between the $8 \mathrm{a}$ and $16 \mathrm{c}$ sites play a crucial role and in the plateau region in the potential profile two structural phases coexist. ${ }^{27}$ Recently, operando EELS on Li $\mathrm{K}$ edge combined with first-principle calculations identified a metastable state, which consists of distorted Li polyhedra, and is proposed to play an essential role to mediate $\mathrm{Li}^{+}$-ion migration. ${ }^{21}$

The success of the LTO material for repeated cycles of charge and discharge arises from the small lattice constant change between the two LTO phases. This is its biggest advantage over alternative intercalating anode systems because it allows rapid charging and many cycles. As we will show, the small change in the lattice parameter is significant enough to cause apparent patterns of strain in micron-sized grains of LTO with a sufficiently sensitive measurement, such as our in situ Bragg coherent diffraction imaging (BCDI) probe a single LTO particle presented here. We observe the development of the local displacement field in 3D by BCDI under in situ conditions while discharging the LTO anode material. BCDI is a lensless imaging technique and, with a suitable choice of iterative algorithms, allows one to get three-dimensional shapes of the nanocrystals as complex $3 \mathrm{D}$ density maps. ${ }^{28}$ In the images, the amplitude represents the ordered electron density of the particle, and the $3 \mathrm{D}$ phase distribution, $\varphi(r)$, is due to local displacements in strained nanocrystals. The mapping is fully quantitative and given by the relationship $\varphi(r)=\mathbf{Q} \cdot \mathbf{u}(\mathbf{r})$, where $\mathbf{Q}$ is the momentum transfer vector of the reciprocal lattice point measured and $\mathbf{u}(\mathbf{r})$ is the vector displacement field representing the distortions from the ideal crystal lattice. ${ }^{29}$ The spatial derivative of the displacement field is a component of the formal strain tensor. Since only one component of the displacement field is measured at each Bragg reflection, phase images from three or more independent $\mathbf{Q}$-vectors must be combined to obtain $3 \mathrm{D}$ spatial reconstruction of the vector field $\mathbf{u}(\mathbf{r})$. As we report here, the small difference in the lattice parameter between the two structures is sufficient to produce an observable local displacement field: if side-by-side regions of $\mathrm{Li}_{4} \mathrm{Ti}_{5} \mathrm{O}_{12}$ to $\mathrm{Li}_{7} \mathrm{Ti}_{5} \mathrm{O}_{12}$ were to be present within the $800 \mathrm{~nm}$ LTO nanoparticle, there would be a phase difference of 0.89 rad, using the lattice parameters reported in ref 17 (details of derivation is given in the SI). This provides the BCDI method with plenty of sensitivity to observe nanoscale displacement fields in these materials.

In this manuscript, we present $3 \mathrm{D}$ renderings of phase transformation of the lithium titanate (LTO) anode material during discharging, obtained using the BCDI technique. Inverted images show the formation of nanodomains with a size of about $200 \mathrm{~nm}$ as positively and negatively shifted image phases in the reconstructed image in the two-phase region of the discharging cycle. This result is an experimental confirmation of the domain model proposed on the same material. ${ }^{30}$ A slice through the rendered inverted images shows displacement field inhomogeneities in the sample but does not show dislocations. In addition, the total $d$-spacing change estimated in a fully discharged single LTO particle is much larger than the bulk.

\section{MATERIALS AND METHODS}

Sample Preparation. The samples for the in situ BCDI experiment were prepared using the conventional 2032-type coin cells. The $\mathrm{Li}_{4} \mathrm{Ti}_{5} \mathrm{O}_{12}$ (LTO) active material from Primet Precision Materials, Inc. was mixed with a conductive agent (Super P conductive carbon black) and poly(vinylidene fluoride) (PVDF) binder in $N$-methyl-2-pyrrolidone (NMP) solvent with a 2:1:1 ratio, which was further coated on the carbon paper current collector and dried in a vacuum oven. The thin LTO electrode was then punched into a $10 \mathrm{~mm}$ disk and assembled in an Ar-filled glove box with the $\mathrm{Li}$ metal as the counter electrode and a Clegrad polypropylene (PP) separator. The electrolyte was prepared by mixing a $1.0 \mathrm{M}$ lithium hexafluorophosphate $\left(\mathrm{LiPF}_{6}\right)$ solution in ethylene carbonate $\left(\left(\mathrm{CH}_{2} \mathrm{O}\right)_{2} \mathrm{CO}\right)$ :dimethyl carbonate $\left(\mathrm{C}_{3} \mathrm{H}_{6} \mathrm{O}_{3}\right)$, with a $1: 1$ volume ratio. For the in situ characterization, an $\mathrm{X}$-ray-transparent Kapton window was fabricated in the stainless-steel case by sealing it with epoxy. Before the coin cell was assembled, we characterized the density and morphology of the electrodes with a scanning electron microscope (SEM) (Figure 1C). The SEM result shows faceted 400$800 \mathrm{~nm}$ sized LTO particles with a characteristic elongated ellipsoidal 


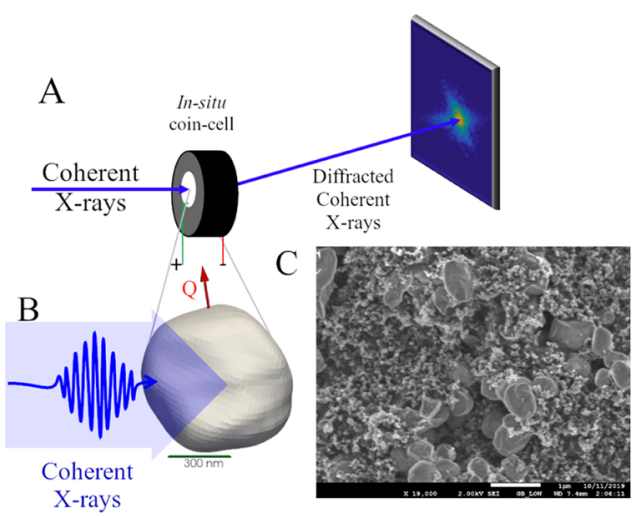

Figure 1. Experimental setup of the in situ Bragg coherent diffraction imaging (BCDI). (A) In situ BCDI setup at 34-ID-C beamline, APS. (B) Three-dimensional reconstructed image of a single LTO crystal, shown using isosurface rendering representation. For the reconstruction, two-dimensional coherent diffraction patterns were collected by rocking the sample in and out off the Bragg condition. The arrow labeled as $Q$ is the scattering vector. (C) SEM images of newly prepared LTO crystals reveal a clear facetted morphology of the specimen. The white scale bar is $1 \mathrm{~mm}$.

shape, which are ideal for BCDI experiments. We assembled several coin cells in the Argon glove box.

In Situ Powder X-ray Diffraction (XRD). In situ X-ray diffraction data were collected at the 28-ID-2 XPD beamline of the National Synchrotron Light Source (NSLS-II). The in situ X-ray diffraction (XRD) testing electrodes were prepared by coating the electrode slurry, a mixture of LTO, conductive carbon black, and poly(vinylidene fluoride) with a 8:1:1 volume ratio, on a current aluminum collector. The experiment under in situ conditions was conducted using the $67 \mathrm{keV}(\lambda=0.185 \AA) \mathrm{X}$-ray beam $0.5 \times 0.5 \mathrm{~mm}^{2}$ in size. X-ray diffraction patterns were acquired using a $2 \mathrm{D}$ PerkinElmer detector $\left(2048 \times 2048\right.$ pixels with $200 \mathrm{~mm}^{2}$ pixel size) located $1.49 \mathrm{~m}$ from the sample, calibrated using $\mathrm{CeO}_{2}$ standard powder. The $2 \mathrm{D}$ diffraction images were integrated azimuthally using pyFAI and GSAS-II was used for Rietveld refinement. ${ }^{31,32}$

In Situ Bragg Coherent Diffraction Imaging. Bragg coherent diffraction data were collected at the 34-ID-C beamline of the Advanced Photon Source (APS) (see Figure 1). For the coherent diffraction analysis shown in this manuscript, we used 11.2 and $9 \mathrm{keV}$ monochromatic beams in two independent experiments. The coherent X-ray beam was focused using a pair of Kirkpatrick-Baez (KB) mirrors to $\sim 1 \times 1 \mu \mathrm{m}^{2}$ illuminating the LTO nanocrystals. As shown in Figure 1A, the measurement was done on a $10 \mu \mathrm{m}$ thick LTO electrode in transmission geometry. We used the same in situ coin-cell setup, which was implemented in the previous experiments ${ }^{33}$ with a window opening of $1 \mathrm{~mm}$. The coin cell was mounted on a coin cell holder vertically with the LTO electrode located downstream to minimize the absorption of the diffracted X-rays. Prior to the experiment, the electrodes were tested via galvanostatic cycling and show a flat plateau region at about $1.5 \mathrm{~V}$ with a sharp voltage reduction in the early discharge. In addition, the coin cells were tested by a multimeter to ensure the open circuit voltage is $1.5 \mathrm{~V}$ (i.e., a fully functional coin cell). The particle density and morphology of the LTO particles were further examined with SEM, shown in Figure 1C, finding 400-800 nm sized LTO particles. From the fringe spacing in the diffraction patterns, we estimated that the measured LTO nanoparticle size is approximately $800 \mathrm{~nm}$. Coherent X-ray diffraction (CXD) patterns were acquired using a Timepix photon-counting detector mounted $D=1.95 \mathrm{~m}$ away from the sample. We obtained full rocking curves around the (400) and (111) Bragg reflections, each in a separate measurement, and collected $2 \mathrm{D}$ CXD patterns using a $2 \mathrm{D}$ detector at two-theta angles of 38.5 and $16.4^{\circ}\left(\Delta \theta= \pm 0.15^{\circ}\right)$, respectively. Although the full sensor of the detector has $512 \times 512$ pixels with $55 \mathrm{~mm}^{2}$ pixel size, the coherent diffraction patterns were collected utilizing just the first quadrant sensor, which has fewer bad pixels. Automatic background subtraction is implemented within the detector. For the (400) set of data, we collected 120 CXD patterns by rocking the sample in $0.0025^{\circ}$ steps around the Bragg peak while we were cycling the coin cell at the $\mathrm{C} / 2$ discharge rate (the $\mathrm{C} / 2$ rate is the current value discharge a battery in $2 \mathrm{~h}$ ). For the second set of data, we collected $60 \mathrm{CXD}$ patterns while rocking the sample in increments of $0.005^{\circ}$ around the (111) Bragg peak while we were cycling the coin cell at the $\mathrm{C} / 2$ discharge rate. For both sets of measurements, the full discharge took roughly $4 \mathrm{~h}$. A full 3D diffraction pattern took about $2 \mathrm{~min}$ to measure. Between consecutive scans, we optimized the sample position on a piezo scanning stage, to maintain the Bragg condition and avoid sample misalignment. The coin cell was discharged using an 8-channel MACCOR battery cycler while the series of measurements progressed.

Phase Retrieval. Before feeding the 3D diffraction data to an iterative phasing algorithm, implemented in Matlab, ${ }^{29,34-37}$ both a white-field correction and a hot pixel removal were applied for each frame of the 3D data stack. For the phase retrieval, we used a total of 620 iterations with 20 iterations of error-reduction (ER), and 180 iterations of a Hybrid-Input-Output (HIO) algorithms, ${ }^{38,39}$ used alternately, with the iteration starting and ending with ER. We used the partial-coherence correction since this was found in the past to improve the reconstructed image quality. ${ }^{34}$ The reconstructed 3D phase images shown throughout this manuscript were shifted with respect to the center phase value for display purposes.

Pearson Cross-Correlation Calculation. The Pearson crosscorrelation coefficient $\left(r_{\mathrm{AB}}\right)$ between diffraction patterns $\mathrm{A}$ and image $\mathrm{B}$ was calculated the following formula

$$
r_{\mathrm{AB}}=\frac{\sum_{i=1}^{n}\left(A_{i}-\bar{A}\right)\left(B_{i}-\bar{B}\right)}{\sqrt{\sum_{i=1}^{n}\left(A_{i}-\bar{A}\right)^{2}} \sqrt{\sum_{i=1}^{n}\left(B_{i}-\bar{B}\right)^{2}}}
$$

Where the sum in $i$ is over the $2 \mathrm{D}$ region of $n$ pixels containing signal, $A_{i}$ and $B_{i}$ are the pixel intensity values, and $\bar{A}=1 / n \sum_{i=1}^{n} A_{i}, \bar{B}=1 / n$ $\sum_{i=1}^{n} B_{i}$ are the mean intensity values of the $2 \mathrm{D}$ diffraction patterns $\mathrm{A}$ and $\mathrm{B}$, taken from the slice at the center of the rocking curve, respectively. The correlation coefficient $r_{\mathrm{AB}}$ by definition ranges from 0 to 1 , where $r_{\mathrm{AB}}=1$ implies a perfect correlation between $\mathrm{A}$ and $\mathrm{B}$ diffraction patterns and $r_{\mathrm{AB}}=0$ means no correlation between them.

Extracting In Situ Single-Particle Lattice Constant Changes. As a first step, we looked at the position of the (400) Bragg peak as a function of discharge voltage. We correlated the voltage cycler timestamp with each rocking scan timestamp. Since the cycler timestamp is relative to the start time, it must be corrected with the known precise starting time. Using these, we obtained the cell voltage readout of each rocking scan. The diffractometer motor positions $\delta, \gamma$, $\chi, \phi$, and $\theta$ are all recorded in a scan file. The detector angles $\gamma$ and $\delta$ are related to the total diffraction $2 \theta$ angle to a very good approximation as

$$
\cos (2 \theta)=\cos (\delta) \cos (\gamma)
$$

We calculated the $\gamma_{i}$ and $\delta_{i}$ angles from the pristine state diffraction peak positions $p_{x 0}, p_{y 0}$ and detector angles $\gamma_{0}, \delta_{0}$ and the diffraction peak positions on the detector $p_{x i} p_{y i}$

$$
\begin{aligned}
& \gamma_{i}=\frac{\left(p_{x i}-p_{x 0}\right) P}{D}\left(\frac{180}{\pi}\right)+\gamma_{0} \\
& \delta_{i}=\frac{\left(p_{y i}-p_{y 0}\right) P}{D}\left(\frac{180}{\pi}\right)+\delta_{0}
\end{aligned}
$$

where $P=55 \mu \mathrm{m}$ is the detector pixel size and $D=1.95 \mathrm{~m}$ is the sample-to-detector distance. Next, we can calculate $2 \theta_{i}$ for each scan using eq 1 and $d$-spacing, $d_{i}$ for each peak using Bragg's law. Finally, we calculate the relative $d$-spacing change, which is $\Delta d / d=\left(d_{i}-d_{\mathrm{o}}\right) /$ $d_{\mathrm{o}}$, where $d_{\mathrm{o}}$ is the $d$-spacing for the pristine state. The quantities calculated using this procedure are shown in Figure 2. 

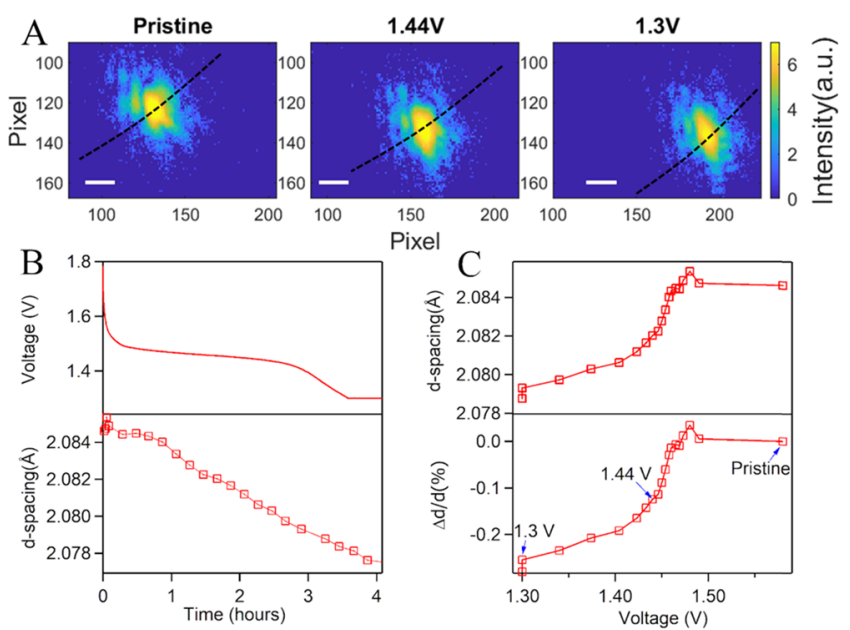

below $1.48 \mathrm{~V}$, we see more lattice contraction. However, the $d$ spacing observed for our $400 \mathrm{~nm}$ particles, $\Delta d / d \cong 0.2 \%$, while larger than the bulk, is smaller than the $\Delta a / a=0.81 \%$ lattice contraction reported in even smaller size nanosized materials. ${ }^{43}$ The lattice parameter changed from $8.3526(1)$ to $8.4203(1) \AA$ and from $8.3585(1)$ to $8.3852(4) \AA$ for the 44 and $120 \mathrm{~nm}$ nanosized samples for $\mathrm{Li}_{4} \mathrm{Ti}_{5} \mathrm{O}_{12}$ and $\mathrm{Li}_{7} \mathrm{Ti}_{5} \mathrm{O}_{12}$ structural phases, respectively. This implies that during a discharge the 44 nm nanosized samples showed $\Delta a / a=0.81 \%$ change, whereas the $120 \mathrm{~nm}$ showed $\Delta a / a=0.32 \%$. A systematic decrease of the lattice contraction effects with increasing particle size is to be expected due to the decrease in the surface area to volume ratio, which is the influence of the interface energy, which would be enhanced in smaller sized LTO samples. We note that density functional theory-based first-principles calculations predicted lattice parameter contraction between $\mathrm{Li}_{4} \mathrm{Ti}_{5} \mathrm{O}_{12}$ and $\mathrm{Li}_{7} \mathrm{Ti}_{5} \mathrm{O}_{12}$ structural phases, ${ }^{44}$ which is much larger than the experimental results. ${ }^{17}$

The measured diffraction pattern intensity is related to the (400) Bragg peak. (A) Logarithm of the diffraction intensity at three different discharge states measured from a single LTO particle. (B) $\mathrm{C} / 2$ discharging cycle of the coin cell for the experiment (upper) and $d$-spacing changes as a function of time extracted from the shift of the (400) diffraction peak on the detector (lower). (C) Extracted lattice $d$-spacing (upper) and relative changes (lower) of the same LTO particle during the discharge cycle as a function of the voltage of the coin cell. The white scale bar is 20 pixels, which corresponds to $\sim 3 \times 10^{-3} \AA^{-1}$.

\section{RESULTS AND DISCUSSION}

(400) Bragg Peak Results. During the in situ BCDI experiments, we cycled the electrode at the $\mathrm{C} / 2$ discharging and charging rate while tracking a single LTO particle. In the past, similar experiments were carried out on battery cathode materials. ${ }^{40,41}$ Figure $2 \mathrm{~A}$ shows an intensity map on the logarithmic scale of the area surrounding one of the (400) coherent diffraction peaks measured from a single LTO particle in its pristine state and at 1.44 and $1.3 \mathrm{~V}$ discharge states. During the discharging process (from left to right shown in Figure 2A), a two-step evolution of the diffraction peak signal was observed: (i) initially, the peak moves inward to a lower $2 \theta$ angle indicating a slight lattice expansion and it moves around the powder ring, which is due to the rotation of the LTO particle, and (ii) in later discharging states, it moves radially outward to a higher $2 \theta$ angle indicating a lattice contraction. In addition, our in situ XRD analysis showed an initial small lattice expansion during the early discharge cycle, followed by a $\sim 0.066 \%$ of lattice contraction shown in Figure S5 of the SI.

In the later stage, $\mathrm{Li}^{+}$migration from the $8 \mathrm{a}$ sites to the $16 \mathrm{c}$ sites leads to lattice contraction. The lattice contraction observed in our bulk XRD measurement is consistent with earlier reports. ${ }^{17}$ The voltage profile of the discharging cycle of the electrode during in situ BCDI measurements and the calculated $d$-spacing changes as a function of time are shown in upper and lower panels of Figure 2B, respectively; the $d$ spacing decreases continuously, with the discharge cycle consistent with lattice contraction. However, the lattice contraction measured in our BCDI experiment is larger than in earlier reports for bulk LTO samples, ${ }^{17}$ where a difference of $\Delta a / a=0.07 \%^{18,42}$ was reported. In top and bottom panels of Figure 2C, we plot the $d$-spacing and $d$-spacing changes with the discharging voltage, respectively. During the early stage of discharge, both the $d$-spacing and $d$-spacing stay constant, but squared amplitude of the Fourier transform of the electron density of the nanocrystal. It is expected that, during the discharging process, $\mathrm{Li}$ ions insert into the LTO particle, which can result in the local displacement field in the particle. To explore this phenomenon, we calculate the Pearson crosscorrelation coefficient between all of the different coherent diffraction patterns done at different discharge voltages with the pristine coherent diffraction pattern. As shown in Figure $3 \mathrm{~A}$, the result highlights the differences between two diffraction

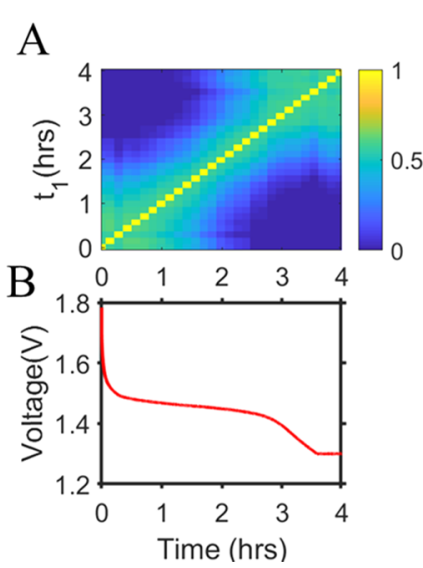

Figure 3. Monitoring structural phase transformation in LTO using the (400) Bragg peak during the $\mathrm{C} / 2$ discharge cycle. (A) Pearson cross-correlation of the (400) diffraction peak between different times during discharge. The correlation is calculated on the central diffraction pattern of the rocking data. (B) Voltage across the coin cell as a function of time.

patterns as changes in the correlation coefficient. Interestingly, the correlation plot shows two distinct regions, the first $2 \mathrm{~h}$ of the discharging process we observe a strong correlation $>0.6$ and the correlation drops appreciably below 0.5 in the last $2 \mathrm{~h}$. Since, the change of the diffraction pattern is the reflection of phase transformation within the scattering volume of the LTO particle. Therefore, the regime between 2.5 to $4 \mathrm{~h}$ of the correlation plot shown in Figure $3 \mathrm{~A}$ is due to the structural transformation of the particle, called the two-phase regime. ${ }^{30}$ This implies that partial uptake of $1 \mathrm{Li}$ ion per unit cell does change the structure locally; however, the diffraction pattern does not change significantly, which is why we get a strong 
correlation for the first $2 \mathrm{~h}$. An uptake of more than one $\mathrm{Li}$ ion per unit cell leads to decorrelation in the diffraction pattern due to a change in the structure as we can see in the correlation plot. Since radiation damage can also change the diffraction pattern, to explore this possibility we measured a single pristine LTO nanocrystal for $2 \mathrm{~h}$ without any voltage cycling. ${ }^{45}$ As shown in Figure S1, this shows strong correlation at all times, which indicated no evident aging or apparent beam damage in the single LTO particle. This implies that the effect observed in Figure $3 \mathrm{~A}$ can only be due to discharge-related changes in the LTO nanocrystal.

(111) Bragg Peak Results. On a different sample, we measured the (111) Bragg peak and calculated the Pearson correlation coefficient. The results in Figure 4A show a clear
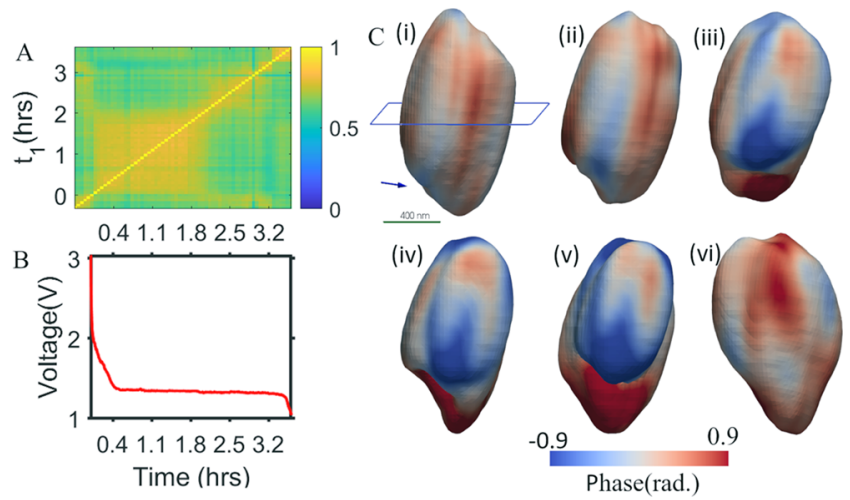

Figure 4. Evidence of the structural phase transformation in LTO during the discharging cycle. (A) Pearson cross-correlation plot of the central (111) diffraction peak of the different discharge states. (B) C/ 2 discharging cycle of the coin cell for the experiment as a function of time. The (i) $-(v i)$ indicated in the curve are points for which the images of the LTO single particles were reconstructed. (C) (i) -(vi) In situ BCDI images of the 3D LTO particle using the isosurface representation, colored by the phase value, measured at $3 \mathrm{~V}$ (pristine), $1.69 \mathrm{~V}(20 \mathrm{~min}), 1.36 \mathrm{~V}(40 \mathrm{~min}), 1.35 \mathrm{~V}(50 \mathrm{~min}), 1.35 \mathrm{~V}(1 \mathrm{~h})$, and $1.3 \mathrm{~V}(3 \mathrm{~h})$. The blue arrow indicated the scattering vector $(Q)$, and the green scale bar is $400 \mathrm{~nm}$. The blue plane shown in the pristine state shows the spatial location of the slices shown in Figure 5.

phase transformation of the LTO sample during the discharge, similar to what was seen for the crystal in Figure 3. Figure 4C shows reconstructed images of the LTO particle, where a clear transformation from a more uniform state to one with a stronger phase variation on its surface. The shape of the crystal was not constrained in the analysis and appears to show some small variations; we interpret this as coupling of the surface displacement field with the isosurface contour level and not a physical change. We attribute this phase variation to the coexistence of $\mathrm{Li}$-poor and $\mathrm{Li}$-rich regions, consistent with previously reported X-ray spectroscopic results. ${ }^{30,46}$ To visualize how the image phase transformation develops inside the LTO particle, we examine slices parallel to the $Q$-vector of the reconstructed images shown in Figure 5. In the early stage of the discharge state (down to $1.44 \mathrm{~V}$ ), there are no significant changes in the image phase variation within the crystal. However, below $1.35 \mathrm{~V}$ (after $1 \mathrm{~h}$ ), there is a build-up of different sets of "nanodomains" seen as positively (red) and negatively (blue) shifted regions. Since this is the discharge stage where the sample is expected to be in transition between the two LTO phases, ${ }^{47}$ these nanodomains are attributed to $\mathrm{Li}^{-}$ rich and Li-poor regions within a single nanocrystal. It appears that an intense image phase difference develops on one side of the LTO particle and propagates into the internal part of the particle. Although the observed image phase change is a consequence of $\mathrm{Li}$ insertion, our observation does not provide direct evidence of $\mathrm{Li}$-ion migration, for example, whether $\mathrm{Li}$ enters the core of the particle or remains confined to the outer edges. However, since the diffusion coefficient, $D$, of $\mathrm{Li}^{+}$ions in LTO is $10^{-11} \mathrm{~cm}^{2} / \mathrm{s}$, ${ }^{48}$ the average distance that the $\mathrm{Li}^{+}$ions can travel in the LTO material, calculated using Fick's law $\left\langle x^{2}\right\rangle$ $=4 \mathrm{D} \tau$, where $\tau$ is the diffusion time, is $2 \mu \mathrm{m}$ in $1 \mathrm{~h}$. Because this distance is larger than the nanoparticles we are measuring, this means that the $\mathrm{Li}^{+}$ions should invade the whole LTO nanocrystal within an hour, and the changes we observe are then due to the local $\mathrm{Li}$ distribution.

To quantify the displacement field inside the reconstructed images, we calculated the standard deviation of the image phase over a range of different volumes selected in the center of the particle as a function of the discharge voltage, as shown in Figure 5C. Similar trends during discharge are seen for all of the choices of volume. The significant increase of standard deviation starting in the middle of the discharge plateau quantifies the appearance of inhomogeneous displacement fields within the particle, which is presumably due to the coexistence of $\mathrm{Li}_{4} \mathrm{Ti}_{5} \mathrm{O}_{12}$ and $\mathrm{Li}_{7} \mathrm{Ti}_{5} \mathrm{O}_{12}$ regions within the sample.

At its maximum, there is about 0.4 radians of inhomogeneity seen in the images, extending over regions of $\sim 100 \mathrm{~nm}$ lateral dimension. This is consistent with the displacement field expected from side-by-side domains of the two material phases, estimated in the Introduction of this paper. A 3D image is shown in Figure $6 \mathrm{~A}$ in the middle of the inhomogeneous discharge state. The gray-colored background uses a low contour level to highlight the particle shape. Two clear domains with a gap in between can be seen at a higher amplitude contouring level with their surface image phases coded using the color scheme shown. The apparent gap between the domains is due to the finite resolution and the chosen contour level. The phase difference of $1.12 \mathrm{rad}$ between the domains corresponds to a lateral shift (along the $Q$-vector) of about $1 / 6$ of a (111) $d$-spacing, as illustrated by the atomic models shown in Figure 6B.

\section{CONCLUSIONS AND OUTLOOK}

We studied the structural phase transformation of the anode material $\mathrm{Li}_{4} \mathrm{Ti}_{5} \mathrm{O}_{12}$ during its lithiation process using in situ BCDI measurements. When observing the (400) Bragg peak of a single LTO particle, we observed more than a $0.2 \%$ change in the $d$-spacing when comparing the pristine and fully lithiated states, which is significantly larger than previously reported results from powder XRD experiments. ${ }^{14,15,30,49}$ This apparent discrepancy must be considered in the context of each measurement: the powder XRD yields the ensemble average lattice constant change of an ensemble average of a large volume of the anode material, whereas the BCDI data result from a single LTO nanocrystal. Nanoparticles are well-known to respond differently from the bulk material due to their larger relative surface area. The two-time correlation plots of the recorded coherent $\mathrm{X}$-ray diffraction patterns demonstrate a clear structural evolution during the lithiation process. BCDI reconstruction, around the middle of the discharge plateau, shows that the onset of $\mathrm{Li}$ insertion is found to lead to a heterogeneous image phase distribution extending throughout the volume of the nanocrystal, which disappears again as the 

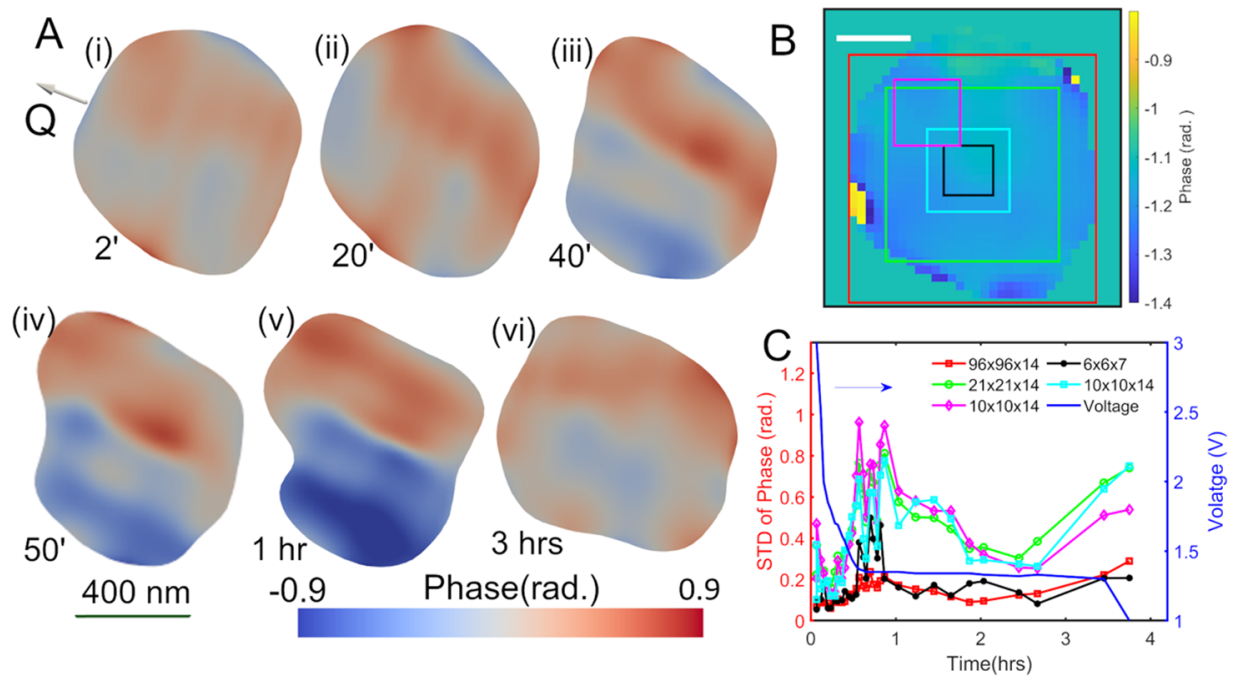

Figure 5. (A) (i) -(vi) Slices of the in situ BCDI reconstructed image from its (111) Bragg peak at 3, 1.69, 1.36, 1.35, 1.35, and 1.3 V, respectively, to show the internal displacement field development in the particle and approximate time of the measurement is also indicated. The biggest image phase variation and relative image phase change is clear in the plateau region of the discharging voltage. (B) Central $x y$-slice of the reconstructed image (in the detector coordinates) with the selected voxel volumes shown as rectangles $96 \times 96 \times 14$ (red), $21 \times 21 \times 14($ green), $10 \times 10 \times 14$ (cyan), $6 \times 6 \times 7$ (magenta), and $10 \times 10 \times 14$ (black) measured at different discharge time. The white scale bar shown is 10 voxels and each voxel is about $(25 \mathrm{~nm})^{3}$. (C) Calculated standard deviations (STD) and the discharge voltages for the corresponding different voxel volumes of the reconstructed image.

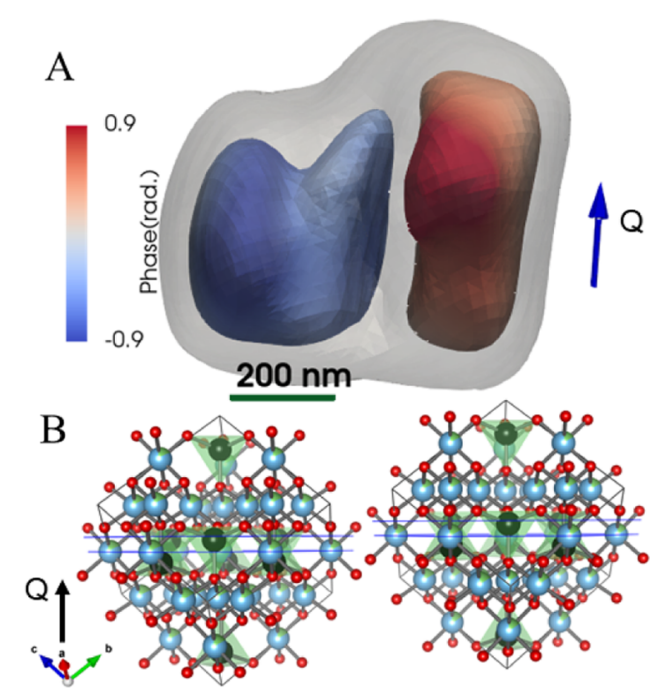

Figure 6. Material phase separation and relative image phase change in the two-phase stage. (A) Reconstructed (111) LTO single-particle image at $1.35 \mathrm{~V}$, resulting in two phase-domains in the single LTO particle due to material phase separation. (B) Relative image phase change is $1.12 \mathrm{rad}$, which corresponds to the $0.8 \AA$ translation along the $Q$-vector shown, which is equal to the $\mathrm{Li}$ - $\mathrm{Ti}$ plane separation shown.

discharge completes. This informs us the formation of a lateral domain structure, implying a nonuniform lithium concentration between nanodomain regions lying side-by-side within the nanocrystal, assumed to have the $\mathrm{Li}_{4} \mathrm{Ti}_{5} \mathrm{O}_{12}$ and $\mathrm{Li}_{7} \mathrm{Ti}_{5} \mathrm{O}_{12}$ compositions. The reconstructed image in Figure 6 in the middle of the voltage plateau region shows a $2 \pi / 6$ image phase change between the two spatially distinct regions of the single LTO nanocrystal. This image phase shift corresponds to $d / 6$ $(=0.8 \AA)$ rigid-body shift between the two regions, corresponding to a Li-Ti layer spacing, illustrated in Figure 6B.
By monitoring the standard deviation of the image phase, we observed the biggest image variation in the middle of the plateau of the discharge where the transformation from $\mathrm{Li}_{4} \mathrm{Ti}_{5} \mathrm{O}_{12}$ and $\mathrm{Li}_{7} \mathrm{Ti}_{5} \mathrm{O}_{12}$ would be half-completed. The heterogeneous local displacement field distribution permeating the LTO nanocrystal investigated was seen to build up during discharge and then taper off at the end of the cycle. At no point in any of our in situ BCDI experiments did we detect the presence of any crystal defects, such as dislocations, which are commonly observed in other battery material systems. ${ }^{40,41}$ Dislocation-mediated transformations are not expected to reverse perfectly upon charge cycling and would be expected to lead eventually to crystal degradation processes. Both our observations of lateral strain domain formation and the absence of mobile dislocations, with Burgers vector accessible in our experimental geometry, imply that the LTO crystal lattice remains completely intact during the charge-discharge cycle. This may explain the excellent cycling stability of LTO over other battery materials, which makes it suitable for durable, long-life batteries.

\section{ASSOCIATED CONTENT}

\section{(1) Supporting Information}

The Supporting Information is available free of charge at https://pubs.acs.org/doi/10.1021/acsaem.0c02010.

Checking X-ray beam damage, two-phase transformation of the LTO particle, displacement projected along $Q$ vector, lattice constant gradient, and in situ X-ray diffraction results (PDF)

\section{AUTHOR INFORMATION}

\section{Corresponding Authors}

Tadesse A. Assefa - Condensed Matter Physics and Materials Science Department, Brookhaven National Laboratory, Upton, New York 11793, United States; (1) orcid.org/00000003-3904-0846; Email: tassefa@bnl.gov 
Ian K. Robinson - Condensed Matter Physics and Materials Science Department, Brookhaven National Laboratory, Upton, New York 11793, United States; London Centre for Nanotechnology, University College London, London WC1E 6BT, U.K.; Email: irobinson@bnl.gov

\section{Authors}

Ana F. Suzana - Condensed Matter Physics and Materials Science Department, Brookhaven National Laboratory, Upton, New York 11793, United States

Longlong Wu - Condensed Matter Physics and Materials Science Department, Brookhaven National Laboratory, Upton, New York 11793, United States

Robert J. Koch - Condensed Matter Physics and Materials Science Department, Brookhaven National Laboratory, Upton, New York 11793, United States; 이이.org/00000003-4995-2101

Luxi Li - Advanced Photon Source, Argonne National Laboratory, Argonne, Illinois 60439, United States

Wonsuk Cha - Advanced Photon Source, Argonne National Laboratory, Argonne, Illinois 60439, United States

Ross J. Harder - Advanced Photon Source, Argonne National Laboratory, Argonne, Illinois 60439, United States

Emil S. Bozin - Condensed Matter Physics and Materials Science Department, Brookhaven National Laboratory, Upton, New York 11793, United States

Feng Wang - Condensed Matter Physics and Materials Science Department, Brookhaven National Laboratory, Upton, New York 11793, United States; 10 orcid.org/0000-0003-40689212

Complete contact information is available at: https://pubs.acs.org/10.1021/acsaem.0c02010

\section{Author Contributions}

T.A.A., F.W., and I.K.R. designed the research project, T.A.A., L.W., A.S., W.C., L.X.L., R.H., F.W., and I.K.R. conducted the BCDI experiments at the 34-ID-C, APS, T.A.A. and I.K.R. analyzed the BCDI data, and R.K. analyzed the XRD data with inputs from E.S.B. The manuscript was written by T.A.A. and I.K.R., with contributions from all authors. All authors have given approval to the final version of the manuscript.

\section{Notes}

The authors declare no competing financial interest.

\section{ACKNOWLEDGMENTS}

We are very grateful for the help of Yao Shanshan with the sample preparation and help with in situ XRD measurements done at NSLS-II, 28-ID-2 beamline. We are also very grateful for the help of Jaiming Bai for the help with in situ XRD measurements done at NSLS-II, 28-ID-2 beamline. T.A.A. would like to thank Xiaojing Huang for his comments on the experimental results. The work at the Brookhaven National Laboratory was supported by the U.S. Department of Energy (DOE), Office of Basic Energy Sciences, Division of Materials Sciences and Engineering, under Contract no. DE-SC0012704. The sample characterization used the resources of the Centre for Functional Nanomaterials, which is a U.S. DOE Office of Science Facility, at the Brookhaven National Laboratory under Contract no. DE-SC0012704. F.W. acknowledges the support of the U.S. Department of Energy, Office of Energy Efficiency and Renewable Energy, Vehicle Technologies Office, under Contract no. DE-SC0012704. This research used the 28-ID-I
(XPD) beamline of the National Synchrotron Light Source II, DOE Office of Science User Facilities operated for the DOE Office of Science by the Brookhaven National Laboratory under Contract no. DE-SC0012704. The experiments were carried out at Advanced Photon Source (APS) beamline 34ID-C, and the APS was supported by the U.S. Department of Energy, Office of Science, Office of Basic Energy Sciences, under Contract no. DE-AC02-06CH11357. The beamline 34ID-C was built with U.S. National Science Foundation Grant no. NSF-DMR 9724294 Development of a Coherent X-Ray Diffraction Instrument.

\section{REFERENCES}

(1) Whittingham, M. S. Electrical Energy Storage and Intercalation Chemistry. Science 1976, 192, 1126-1127.

(2) Whittingham, M. S.; Gamble, F. R. The Lithium Intercalates of the Transition Metal Dichalcogenides. Mater. Res. Bull. 1975, 10, 363-371.

(3) Whittingham, M. S. The Role of Ternary Phases in Cathode Reactions. J. Electrochem. Soc. 1976, 123, 315.

(4) Yi, T.-F.; Yang, S.-Y.; Xie, Y. Recent Advances of $\mathrm{Li}_{4} \mathrm{Ti}_{5} \mathrm{O}_{12}$ as a Promising next Generation Anode Material for High Power LithiumIon Batteries. J. Mater. Chem. A 2015, 3, 5750-5777.

(5) Takehara, Z. I.; Kanamura, K. Historical Development of Rechargeable Lithium Batteries in Japan. Electrochim. Acta 1993, 38, 1169-1177.

(6) Whittingham, M. S. Lithium Batteries and Cathode Materials. Chem. Rev. 2004, 104, 4271-4302.

(7) Liu, K.; Pei, A.; Lee, H. R.; Kong, B.; Liu, N.; Lin, D.; Liu, Y.; Liu, C.; Hsu, P.; Bao, Z.; Cui, Y. Lithium Metal Anodes with an Adaptive "Solid-Liquid" Interfacial Protective Layer. J. Am. Chem. Soc. 2017, 139, 4815-4820.

(8) Qian, J.; Xu, W.; Bhattacharya, P.; Engelhard, M.; Henderson, W. A.; Zhang, Y.; Zhang, J. G. Dendrite-Free Li Deposition Using Trace-Amounts of Water as an Electrolyte Additive. Nano Energy 2015, 15, 135-144.

(9) Bai, P.; Guo, J.; Wang, M.; Kushima, A.; Su, L.; Li, J.; Brushett, F. R.; Bazant, M. Z. Interactions between Lithium Growths and Nanoporous Ceramic Separators. Joule 2018, 2, 2434-2449.

(10) Besenhard, J. O.; Yang, J.; Winter, M. Will Advanced LithiumAlloy Anodes Have a Chance in Lithium-Ion Batteries? J. Power Sources 1997, 68, 87-90.

(11) Brousse, T.; et al. Thin-Film Crystalline $\mathrm{SnO}_{2}$-Lithium Electrodes. J. Electrochem. Soc. 1998, 145, 1.

(12) Chan, C. K.; Peng, H.; Liu, G.; McIlwrath, K.; Zhang, X. F.; Huggins, R. A.; Cui, Y. High-Performance Lithium Battery Anodes Using Silicon Nanowires. Nat. Nanotechnol. 2008, 3, 31-35.

(13) Shu, J. Study of the Interface between $\mathrm{Li}_{4} \mathrm{Ti}_{5} \mathrm{O}_{12}$ Electrodes and Standard Electrolyte Solutions in 0.0-0.5 V. Electrochem. Solid-State Lett. 2008, 11, A238.

(14) Shu, J. Electrochemical Behavior and Stability of $\mathrm{Li}_{4} \mathrm{Ti}_{5} \mathrm{O}_{12}$ in a Broad Voltage Window. J. Solid State Electrochem. 2009, 13, 15351539.

(15) Ohzuku, T.; Ueda, A.; Yamamoto, N. Zero-Strain Insertion Material of $\mathrm{Li}\left[\mathrm{Li}_{1 / 3} \mathrm{Ti}_{5 / 3} \mathrm{O}_{4}\right]$ for Rechargeable Lithium Cells. J. Electrochem. Soc. 1995, 142, 1431.

(16) Toshiba's SCiB Rechargeable Battery.

(17) Scharner, S.; Weppner, W.; Schmid-Beurmann, P. Evidence of Two-Phase Formation upon Lithium Insertion into the Li1.33Ti1.67O4 Spinel. J. Electrochem. Soc. 1999, 146, 857.

(18) Ziebarth, B.; Klinsmann, M.; Eckl, T.; Elsässer, C. Lithium Diffusion in the Spinel Phase $\mathrm{Li}_{4} \mathrm{Ti}_{5} \mathrm{O}_{12}$ and in the Rocksalt Phase $\mathrm{Li}_{7} \mathrm{Ti}_{5} \mathrm{O}_{12}$ of Lithium Titanate from First Principles. Phys. Rev. B 2014, 89, No. 174301.

(19) Zhang, W.; Topsakal, M.; Cama, C.; Pelliccione, C. J.; Zhao, H.; Ehrlich, S.; Wu, L.; Zhu, Y.; Frenkel, A. I.; Takeuchi, K. J.; Takeuchi, E. S.; Marschilok, A. C.; Lu, D.; Wang, F. Multi-Stage Structural Transformations in Zero-Strain Lithium Titanate Unveiled 
by in Situ X-Ray Absorption Fingerprints. J. Am. Chem. Soc. 2017, 139, 16591-16603.

(20) Wang, F.; Wu, L.; Ma, C.; Su, D.; Zhu, Y.; Graetz, J. Excess Lithium Storage and Charge Compensation in Nanoscale $\mathrm{Li}_{4+x} \mathrm{Ti}_{5} \mathrm{O}_{12}$. Nanotechnology 2013, 24 (42), 424006.

(21) Zhang, W.; Seo, D.-H. H.; Chen, T.; Wu, L.; Topsakal, M.; Zhu, Y.; Lu, D.; Ceder, G.; Wang, F. Kinetic Pathways of Ionic Transport in Fast-Charging Lithium Titanate. Science 2020, 367, 1030-1034.

(22) Pang, W. K.; Peterson, V. K.; Sharma, N.; Shiu, J.-J.; Wu, S. Lithium Migration in $\mathrm{Li}_{4} \mathrm{Ti}_{5} \mathrm{O}_{12}$ Studied Using in Situ Neutron Powder Diffraction. Chem. Mater. 2014, 26, 2318-2326.

(23) Wilkening, M.; Iwaniak, W.; Heine, J.; Epp, V.; Kleinert, A.; Behrens, M.; Nuspl, G.; Bensch, W.; Heitjans, P. Microscopic Li SelfDiffusion Parameters in the Lithiated Anode Material $\mathrm{Li}_{4+\mathrm{x}} \mathrm{Ti}_{5} \mathrm{O}_{12}(0$ $\leq \mathrm{x} \leq 3)$ Measured by ${ }^{7} \mathrm{Li}$ Solid State NMR. Phys. Chem. Chem. Phys. 2007, 9, 6199-6202.

(24) Hain, H.; Scheuermann, M.; Heinzmann, R.; Wünsche, L.; Hahn, H.; Indris, S. Study of Local Structure and Li Dynamics in $\mathrm{Li}_{4+\mathrm{x}} \mathrm{Ti}_{5} \mathrm{O}_{12}(0 \leq \mathrm{x} \leq 5)$ Using ${ }^{6} \mathrm{Li}$ and ${ }^{7} \mathrm{Li}$ NMR Spectroscopy. Solid State Nucl. Magn. Reson. 2012, 42, 9-16.

(25) Lee, H. H.; Wang, Y. Y.; Wan, C. C.; Yang, M. H.; Wu, H. C.; Shieh, D. T. The Function of Vinylene Carbonate as a Thermal Additive to Electrolyte in Lithium Batteries. J. Appl. Electrochem. 2005, 35, 615-623.

(26) Kitta, M.; Akita, T.; Tanaka, S.; Kohyama, M. Two-Phase Separation in a Lithiated Spinel $\mathrm{Li}_{4} \mathrm{Ti}_{5} \mathrm{O}_{12}$ Crystal as Confirmed by Electron Energy-Loss Spectroscopy. J. Power Sources 2014, 257, 120125.

(27) Ozaki, H.; Tada, K.; Kiyobayashi, T. Monte-Carlo Simulation Combined with Density Functional Theory to Investigate the Equilibrium Thermodynamics of Electrode Materials: Lithium Titanates as Model Compounds. Phys. Chem. Chem. Phys. 2019, 21, $15551-15559$.

(28) Miao, J.; Ishikawa, T.; Robinson, I. K.; Murnane, M. M. Beyond Crystallography: Diffractive Imaging Using Coherent $\mathrm{x}$-Ray Light Sources. Science 2015, 348, 530-535.

(29) Robinson, I.; Harder, R. Coherent X-Ray Diffraction Imaging of Strain at the Nanoscale. Nat. Mater. 2009, 8, 291-298.

(30) Zhang, W.; Topsakal, M.; Cama, C.; Pelliccione, C. J.; Zhao, H.; Ehrlich, S.; Wu, L.; Zhu, Y.; Frenkel, A. I.; Takeuchi, K. J.; Takeuchi, E. S.; Marschilok, A. C.; Lu, D.; Wang, F. Multi-Stage Structural Transformations in Zero-Strain Lithium Titanate Unveiled by in Situ X-Ray Absorption Fingerprints. J. Am. Chem. Soc. 2017, 139, 16591-16603.

(31) Kieffer, J.; Karkoulis, D. PyFAI, a Versatile Library for Azimuthal Regrouping. J. Phys. Conf. Ser. 2013, No. 202012.

(32) Toby, B. H.; Von Dreele, R. B. GSAS-II: The Genesis of a Modern Open-Source All Purpose Crystallography Software Package. J. Appl. Crystallogr. 2013, 46, 544-549.

(33) Li, L.; Xie, Y.; Maxey, E.; Harder, R. Methods for Operando Coherent X-Ray Diffraction of Battery Materials at the Advanced Photon Source. J. Synchrotron Radiat. 2019, 26, 220-229.

(34) Clark, J. N.; Huang, X.; Harder, R.; Robinson, I. K. HighResolution Three-Dimensional Partially Coherent Diffraction Imaging. Nat. Commun. 2012, 3, No. 993.

(35) Yang, W.; Huang, X.; Harder, R.; Clark, J. N.; Robinson, I. K.; Mao, H. K. Coherent Diffraction Imaging of Nanoscale Strain Evolution in a Single Crystal under High Pressure. Nat. Commun. 2013, 4, No. 1680.

(36) Pfeifer, M. A.; Williams, G. J.; Vartanyants, I. A.; Harder, R.; Robinson, I. K. Three-Dimensional Mapping of a Deformation Field inside a Nanocrystal. Nature 2006, 442, 63-66.

(37) Assefa, T. A.; Cao, Y.; Diao, J.; Harder, R. J.; Cha, W.; Kisslinger, K.; Gu, G. D.; Tranquada, J. M.; Dean, M. P. M.; Robinson, I. K. Scaling Behavior of Low-Temperature Orthorhombic Domains in the Prototypical High-Temperature Superconductor $\mathrm{La}_{1.875} \mathrm{Ba}_{0.125} \mathrm{CuO}_{4}$. Phys. Rev. B 2020, 101, No. 054104.
(38) Fienup, J. R. Phase Retrieval Algorithms: A Comparison. Appl. Opt. 1982, 21, 2758.

(39) Saxton, R. W.; Gershberg, W. O. Practical Algorithm for the Determination of Phase From Image and Diffraction Plane Pictures. Optik 1972, 35, 237-250.

(40) Ulvestad, A.; Singer, A.; Cho, H.-M.; Clark, J. N.; Harder, R.; Maser, J.; Meng, Y. S.; Shpyrko, O. G. Single Particle Nanomechanics in Operando Batteries via Lensless Strain Mapping. Nano Lett. 2014, $14,5123-5127$

(41) Ulvestad, A.; Singer, A.; Clark, J. N.; Cho, H. M.; Kim, J. W.; Harder, R.; Maser, J.; Meng, Y. S.; Shpyrko, O. G. Topological Defect Dynamics in Operando Battery Nanoparticles. Science 2015, 348, $1344-1347$.

(42) Colbow, K. M.; Dahn, J. R.; Haering, R. R. Structure and Electrochemistry of the Spinel Oxides $\mathrm{LiTi}_{2} \mathrm{O}_{4}$ and $\mathrm{Li}_{4 / 3} \mathrm{Ti}_{5 / 3} \mathrm{O}_{4} . J$. Power Sources 1989, 26, 397-402.

(43) Yu, X.; Pan, H.; Wan, W.; Ma, C.; Bai, J.; Meng, Q.; Ehrlich, S. N.; Hu, Y.-S.; Yang, X.-Q. A Size-Dependent Sodium Storage Mechanism in $\mathrm{Li}_{4} \mathrm{Ti}_{5} \mathrm{O}_{12}$ Investigated by a Novel Characterization Technique Combining in Situ X-Ray Diffraction and Chemical Sodiation. Nano Lett. 2013, 13, 4721-4727.

(44) Ouyang, C. Y.; Zhong, Z. Y.; Lei, M. S. Ab Initio Studies of Structural and Electronic Properties of $\mathrm{Li}_{4} \mathrm{Ti}_{5} \mathrm{O}_{12}$ Spinel. Electrochem. Commun. 2007, 9, 1107-1112.

(45) Plumley, R.; Sun, Y.; Teitelbaum, S. W.; Song, S.; Sato, T.; Chollet, M.; Wang, N.; Robert, A.; Fuoss, P. H.; Sutton, M.; Zhu, D. Speckle Correlation as a Monitor of X-Ray Free Electron Laser Induced Crystal Lattice Deformation. J. Synchrotron Radiat. 2019, $1470-1476$

(46) Wang, F.; Wu, L.; Ma, C.; Su, D.; Zhu, Y.; Graetz, J. Excess Lithium Storage and Charge Compensation in Nanoscale $\mathrm{Li}_{4+\mathrm{x}} \mathrm{Ti}_{5} \mathrm{O}_{12}$. Nanotechnology 2013, 24, No. 424006.

(47) Orikasa, Y.; Maeda, T.; Koyama, Y.; Murayama, H.; Fukuda, K.; Tanida, H.; Arai, H.; Matsubara, E.; Uchimoto, Y.; Ogumi, Z. Transient Phase Change in Two Phase Reaction between $\mathrm{LiFePO}_{4}$ and $\mathrm{FePO}_{4}$ under Battery Operation. Chem. Mater. 2013, 25, 10321039.

(48) Colbow, K. M.; Dahn, J. R.; Haering, R. R. Structure and Electrochemistry of the Spinel Oxides $\mathrm{LiTi}_{2} \mathrm{O}_{4}$ and $\mathrm{Li}\left[\mathrm{Li}_{4 / 3} \mathrm{Ti}_{5 / 3} \mathrm{O}_{4}\right]$. J. Power Sources 1989, 26, 397-402.

(49) Yi, T. F.; Shu, J.; Zhu, Y. R.; Zhu, X. D.; Yue, C. B.; Zhou, A. N.; Zhu, R. S. High-Performance $\mathrm{Li}_{4} \mathrm{Ti}_{5-\mathrm{x}} \mathrm{VxO}_{12}(0 \leq \mathrm{x} \leq 0.3)$ as an Anode Material for Secondary Lithium-Ion Battery. Electrochim. Acta 2009, 54, 7464-7470. 